\title{
Elevated serum ferritin levels predict new-onset type 2 diabetes: results from the EPIC-Norfolk prospective study
}

\author{
N. G. Forouhi • A. H. Harding • M. Allison • \\ M. S. Sandhu • A. Welch • R. Luben • S. Bingham • \\ K. T. Khaw • N. J. Wareham
}

Received: 10 November 2006 / Accepted: 22 December 2006 / Published online: 2 March 2007

(C) Springer-Verlag 2007

\begin{abstract}
Aims/hypothesis The aim of this study was to examine the association between baseline body iron stores and new-onset diabetes.

Subjects and methods We studied the association between baseline serum ferritin concentration and type 2 diabetes in 360 clinically incident diabetes cases and 758 controls nested within the EPIC (European Prospective Investigation of Cancer)-Norfolk Cohort Study. Serum ferritin levels were categorised into five groups: sex-specific quartiles of the normal range of ferritin and a group with clinically raised ferritin below levels indicative of haemochromatosis. Results Baseline serum ferritin was higher among cases than control participants (geometric mean: men 96.6 vs $67.8 \mathrm{ng} / \mathrm{ml}$, respectively, $p<0.001$; women 45.9 vs $34.8 \mathrm{ng} /$ $\mathrm{ml}$, respectively, $p=0.005$ ). In analyses adjusted for known risk factors (age, BMI, sex, family history, physical activity, smoking habit) and dietary factors measured by 7-day food
\end{abstract}

N. G. Forouhi $(\varangle) \cdot$ A. H. Harding $\cdot$ M. S. Sandhu •

N. J. Wareham

MRC Epidemiology Unit, Elsie Widdowson Laboratories,

Fulbourn Road,

Cambridge CB1 9NL, UK

e-mail: nita.forouhi@mrc-epid.cam.ac.uk

M. Allison

Addenbrooke's Hospital NHS Trust,

Cambridge, UK

M. S. Sandhu $\cdot$ A. Welch $\cdot$ R. Luben $\cdot$ S. Bingham $\cdot$ K. T. Khaw Department of Public Health and Primary Care,

University of Cambridge,

Cambridge, UK

S. Bingham

Medical Research Council Dunn Human Nutrition Unit,

Cambridge, UK diary, the risk of diabetes was markedly elevated in participants with clinically raised ferritin compared with the lowest quartile (odds ratio [OR] 7.4, 95\% CI 3.5-15.4). Further adjustment for potential confounding by inflammation (C-reactive protein, IL-6 and fibrinogen) had no material impact on the observed association, while adjustment for hepatic enzymes (alanine aminotransferase and $\gamma$ glutamyl transferase) and adiponectin attenuated the magnitude of association, but it remained statistically significant (OR 3.2 [1.3-7.6]).

Conclusions/interpretation Serum ferritin is an important and independent predictor of the development of diabetes. This finding may have important implications for understanding the aetiology of diabetes.

Keywords Ferritin · Hepatic enzymes · Inflammation · Type 2 diabetes

\begin{tabular}{|c|c|}
\hline Abbr & viations \\
\hline ALT & alanine aminotransferase \\
\hline CRP & C-reactive protein \\
\hline EPIC & European Prospective Investigation of Cancer \\
\hline GGT & $\gamma$-glutamyl transferase \\
\hline OR & odds ratio \\
\hline
\end{tabular}

Introduction

The nature of the association between abnormal iron storage and the development of type 2 diabetes among individuals without haemochromatosis remains uncertain. In a case-control study nested in a Finnish cohort, men with high iron stores, as measured by the ratio of transferrin receptors to ferritin, had a 2.4-fold elevated risk of incident diabetes compared with those in the lowest quartile [1]. 
Similarly, in the Nurses' Health Study of apparently healthy women, elevated ferritin concentration and a lower ratio of transferrin receptors to ferritin were associated with increased risk of diabetes, which was independent of known diabetes risk factors and of inflammation as measured by C-reactive protein (CRP) [2]. These two prospective studies reduce doubt about the temporal sequence which had affected previous cross-sectional or case-control studies [3-7], but several questions still remain unanswered.

As ferritin is an acute-phase reactant, it is unclear whether adjustment for one marker of inflammation (CRP) [2] is adequate or whether the association is affected by residual confounding. There is now growing evidence for the association of incident diabetes with both the adipocytokine adiponectin $[8,9]$ and hepatic function as measured by the hepatic enzymes alanine aminotransferase (ALT) and $\gamma$ glutamyl transferase (GGT) [10-13]. However, their association with ferritin has not been widely studied, nor are there any studies that have adjusted for these markers to test the independence of the ferritin-diabetes association. As previous studies did not distinguish between the clinically normal and clinically raised ferritin levels in the top percentile category of ferritin, a potential dose-response effect of ferritin on diabetes incidence has not been studied. Furthermore, to the best of our knowledge, the published literature on prospective studies is limited to only two studies of incident diabetes $[1,2]$ plus a recent French study of the combined endpoint of incident non-diabetic hyperglycaemia ( $n=200$ with impaired fasting glucose) and diabetes $(n=31)$ [14]. Thus confirmation of the findings is warranted in other settings.

The aims of our study were, therefore, to test the prospective association of serum ferritin with clinically incident diabetes in a population-based cohort study including both men and women, and to test whether this association is independent of a comprehensive range of inflammatory markers, as well as tests of hepatic function and adiponectin.

\section{Subjects and methods}

Study design and population The current case-control study is nested in the European Prospective Investigation of Cancer (EPIC) Norfolk cohort that recruited 25,631 volunteers, aged 40-74 years, from general practices in Norwich and surrounding towns in Norfolk between 1993 and 1997, and has been described in detail previously [15]. In brief, it was a population-based cohort study where participants attended for a baseline health check, completed a postal questionnaire at 18 months of follow-up, and attended for a second health check in 1998-2000. At follow-up (mean 5.1 years, SD 1.7 years) there were 417 clinically incident cases of diabetes. Two sets of control participants were chosen: one set was matched for age, sex, general practice and recruitment date $(n=417)$, and the other set was additionally frequency matched for BMI $(n=417)$. For this study we used the combined control participants $(n=834)$. Of the 417 cases and 834 control participants, data on ferritin were missing for 133; thus the final study population for this study included 360 case and 758 control participants with complete data. The study was approved by the local research ethics committee and participants gave written informed consent.

Ascertainment of incident diabetes Individuals with selfreported diabetes at baseline were excluded $(n=845)$. Multiple sources of case ascertainment for new-onset diabetes were as follows: self-report of diabetes from the first and second health check and lifestyle questionnaire; self-report of diabetes-specific medication in either of the two follow-up questionnaires or medication brought to the follow-up health check; or an $\mathrm{HbA}_{1 \mathrm{c}}$ level $>7 \%$ at either the baseline or follow-up health check. In addition, nonEPIC sources of information included listing of any EPICNorfolk participant in the general practice diabetes register, local hospital diabetes register, hospital admissions data at that hospital screened for any diabetes-related admissions among study participants, and Office of National Statistics mortality data with coding for diabetes.

A matrix containing data from the seven sources listed above was drawn up and each record with a positive response in any field was examined to determine whether it qualified for a definition of a new case of diabetes as previously described [16]. A validation exercise of this method of case ascertainment was performed, by reviewing the medical records of case and control participants, in 12 of 35 participating general practices and we found that three sources (general practice registers, $\mathrm{HbA}_{1 \mathrm{c}}$ level and selfreport of diabetes) detected $96 \%$ of all known cases of diabetes [16].

Data collection Clinical examination to a standard protocol was performed as previously described [16]. Anthropometric measurement included height $(\mathrm{m})$, weight $(\mathrm{kg})$ and waist and hip circumference $(\mathrm{cm})$. BMI was calculated as weight/ height ${ }^{2}\left(\mathrm{~kg} / \mathrm{m}^{2}\right)$. A blood test was taken for $\mathrm{HbA}_{1 \mathrm{c}}$ estimation using HPLC on a Bio-Rad Diamat analyser (Bio-Rad, Richmond, CA, USA). Plasma vitamin $\mathrm{C}$ was also measured. A detailed health and lifestyle questionnaire was completed at baseline (1993-1997), at 18 months of follow-up and at the second health check (1998-2000). It included items on demography, personal and family history of diabetes among first-degree relatives, smoking, physical activity, medication and diet [16]. In women, a menstrual/ 
menopausal history was recorded and those with their last menstrual period $\geq 2$ years previously were coded as postmenopausal. Information on occupational and leisuretime physical activity was assembled into a four-point physical activity index [17] and smoking was coded as never, former or current.

At baseline, dietary intake was assessed by a 7-day prospective self-recorded food diary, which asked participants to indicate food content as well as portion size for all food consumed for 7 days. Data were entered using an in-house program DINER (Data into Nutrients for Epidemiological Research) [18]. Nutrient intakes in grams were estimated from the reported food intake and in-house databases [19].

Frozen portions of serum from case and control participants were selected for simultaneous analysis in 2005. Serum ferritin was measured using a two-step time-resolved fluoroimmunoassay with an AutoDELFIA ferritin kit (Wallac Oy, Turku, Finland). Typical between-batch CV values are 5.8 and $6.7 \%$ at 4.6 and $355 \mu \mathrm{g} / \mathrm{l}$, respectively. Serum high-sensitivity CRP and liver enzymes were measured using a Dade Behring Dimension ARx automated clinical chemistry system (Deerfield, IL, USA), with between batch CV values as follows: CRP $5.0 \%$ at $14 \mathrm{mg} / \mathrm{l}, 2.8 \%$ at $120 \mathrm{mg} / \mathrm{l}$; ALT $16.9 \%$ at $35 \mathrm{U} / 1,6.8 \%$ at $470 \mathrm{U} / \mathrm{l}$; GGT $2.9 \%$ at $80 \mathrm{U} / 1,1.7 \%$ at $147 \mathrm{U} / 1$. Serum adiponectin was measured using an AutoDELFIA kit (Wallac Oy) with a two-step time-resolved fluoroimmunoassay, with between-batch CV values of $5.2 \%$ at $3.49 \mu \mathrm{g} / \mathrm{ml}, 6.95 \%$ at $8.85 \mu \mathrm{g} / \mathrm{ml}$ and $11.9 \%$ at $15.8 \mu \mathrm{g} / \mathrm{ml}$.

Statistical analysis We calculated mean (SD) or proportion of baseline clinical and metabolic factors and diabetes risk factors among case and control participants, and tested for differences using $t$ or $\chi^{2}$ tests, respectively. The correlations between serum ferritin, inflammatory factors, liver function tests and adiponectin were examined. Skewed variables, including ferritin, were log (natural) transformed to a normal distribution. Participants were classified into five groups according to their sex-specific serum ferritin concentrations, into quartiles of the normal range of ferritin in control participants (groups 1-4), and into a fifth category of clinically raised ferritin (group 5). We defined raised concentrations of ferritin as $\geq 300 \mathrm{ng} / \mathrm{ml}$ for men and $\geq 150 \mathrm{ng} / \mathrm{ml}$ for women, as previously described in an analysis of the Third National Health and Nutrition Examination Survey [3] and as currently defined by the US National Institutes of Health and National Library of Medicine. These cut-off values are close to the 95th percentile cut-off of $305 \mathrm{ng} / \mathrm{ml}$ in men and $165 \mathrm{ng} / \mathrm{ml}$ in women in the current study. We excluded individuals (two men, three women), in whom serum ferritin was elevated beyond three times this upper limit of the reference range to reduce the possibility of including those with undiagnosed haemochromatosis. We performed a sensitivity analysis with the alternative upper limit cut-points of $200 \mathrm{ng} / \mathrm{ml}$ [20, 21] and $300 \mathrm{ng} / \mathrm{ml}$ in women. This latter cut-point was chosen to give the same cut-off in men and women, as some laboratories currently do not use different cut-points by sex.

To examine the distribution of baseline factors across the five groups of baseline ferritin we tested median levels (and interquartile range), with the Kruskall-Wallis test for differences. In logistic regression analyses the dependent variable was incident diabetes vs no diabetes, and the independent variable of interest was ferritin. We performed an unmatched (unconditional) logistic regression analysis and adjusted all analyses for age, sex and BMI to enable the use of all control participants (two control participants for every case), as the frequency matching on BMI was only performed on one set of control participants. In multivariate analyses we constructed models that adjusted for known diabetes risk factors (family history, physical activity and smoking) and potential confounders (dietary factors, inflammatory markers, hepatic enzymes and adiponectin). In women we tested the effect of menopausal status on risk. Tests for departure from linear trend and interaction were conducted using likelihood ratio tests. All analyses were performed using STATA statistical software (version 8.0; Statacorp, College Station, TX, USA).

\section{Results}

Among 360 case and 758 control participants, the mean ages were similar (62.4 and 62.1 years, SD 8.3), and 58\% were men in each group. At baseline, participants who went on to develop diabetes during follow-up vs those who did not were significantly more obese (BMI 29.8 vs $27.7 \mathrm{~kg} /$ $\mathrm{m}^{2}$, waist circumference 103.1 vs $98.6 \mathrm{~cm}$ among men and $96.3 \mathrm{vs} 85.7 \mathrm{~cm}$ among women) and more likely to have a positive family history of diabetes (20.3 vs $9.9 \%$ ), all $p<0.001$. Case participants were less physically active than control participants (inactive: 45.8 vs $36.5 \%$; moderately inactive: 23.9 vs $24.4 \%$; moderately active: 17.8 vs $18.6 \%$, and active 12.5 vs $20.5 \%, p=0.003$ ). Baseline serum ferritin was higher among incident case participants than in control participants in both men (geometric means: 96.6 [95\% CI $84.7-110.2]$ vs 67.8 [95\% CI 62.6-73.5] ng/ml in case and control participants, respectively) and women $(45.9$ [95\% CI $38.1-55.3$ ] vs 34.8 [95 \% CI 31.6-38.4] ng/ml). There was no evidence of a statistical interaction between ferritin levels and sex $(p=0.51)$, and analyses were performed in men and women combined. Table 1 shows the range of ferritin concentrations among men and women in the five ferritin groups that comprise quartiles in the normal range (ferritin, groups 1-4) and a fifth category of clinically 
Table 1 The distribution of biochemical and dietary factors across sex-specific quartiles of ferritin in the normal range and in the clinically raised ferritin category: the EPIC diabetes nested case-control study (1993-2000)

\begin{tabular}{|c|c|c|c|c|c|c|}
\hline & \multicolumn{5}{|l|}{ Ferritin group } & \multirow[t]{2}{*}{$p$ value } \\
\hline & $\begin{array}{l}\text { Group 1 } \\
\text { (quartile 1) }\end{array}$ & $\begin{array}{l}\text { Group } 2 \\
\text { (quartile 2) }\end{array}$ & $\begin{array}{l}\text { Group } 3 \\
\text { (quartile 3) }\end{array}$ & $\begin{array}{l}\text { Group } 4 \\
\text { (quartile 4) }\end{array}$ & $\begin{array}{l}\text { Group } 5 \\
\text { (raised ferritin) }\end{array}$ & \\
\hline \multicolumn{7}{|l|}{ Ferritin (ng/ml) } \\
\hline Men & $<39.4$ & $39.5-71.1$ & $71.2-112.4$ & 112.5 to $<300$ & $\geq 300$ and $<900$ & \\
\hline Women & $<20.6$ & $20.7-36.7$ & $36.8-63.0$ & 63.1 to $<150$ & $\geq 150$ and $<450$ & \\
\hline \multicolumn{7}{|l|}{ Biochemical factors } \\
\hline CRP (mg/dl) & $4.1(2.8-5.7)$ & $4.2(3.0-5.9)$ & $4.2(2.9-6.2)$ & $4.5(3.1-6.6)$ & $4.7(3.0-7.0)$ & 0.11 \\
\hline Fibrinogen $(\mathrm{g} / \mathrm{l})$ & $3.0(2.4-3.5)$ & $3.1(2.6-3.5)$ & $3.0(2.6-3.5)$ & $3.0(2.5-3.4)$ & $2.7(2.3-3.5)$ & 0.13 \\
\hline IL-6 (pg/ml) & $1.1(0.8-2.1)$ & $1.1(0.8-2.1)$ & $1.3(0.8-2.3)$ & $1.3(0.8-2.4)$ & $1.3(0.8-2.6)$ & 0.11 \\
\hline $\begin{array}{l}\text { Plasma vitamin } C \\
(\mu \mathrm{mol} / \mathrm{l})\end{array}$ & $50.0(38.0-61.0)$ & $50.0(36.0-61.0)$ & $52.0(37.0-64.0)$ & $47.0(32.0-59.0)$ & $45.0(26.0-57.0)$ & 0.016 \\
\hline ALT (U/l) & $13.1(11.3-15.5)$ & $13.5(10.6-16.8)$ & $14.4(11.4-17.9)$ & $15.1(12.1-19.0)$ & $19.5(13.5-31.2)$ & 0.0001 \\
\hline GGT (U/1) & $31.0(26.0-40.0)$ & $36.0(26.0-49.0)$ & $36.0(27.0-51.0)$ & $41.0(29.0-60.0)$ & $60.0(38.0-77.0)$ & 0.0001 \\
\hline Adiponectin (ng/ml) & $6.5(4.5-9.4)$ & $6.5(4.4-9.1)$ & $6.5(4.2-9.1)$ & $5.9(4.0-8.4)$ & $4.6(2.8-7.1)$ & 0.0003 \\
\hline \multicolumn{7}{|l|}{ Dietary factors } \\
\hline Total energy (kJ/day) & $7,915(6,883-9,423)$ & $8,293(7,161-9,817)$ & $7,996(6,550-9,730)$ & $8,031(6,587-9,699)$ & $7,635(6,650-10,567)$ & 0.35 \\
\hline Alcohol (g/day) & $2.1(0-8.2)$ & $4.1(0-14.6)$ & $5.3(0-16.3)$ & $7.5(0.5-20.4)$ & $5.3(0-17.9)$ & 0.0001 \\
\hline Red meat (g/day) & $45.5(17.1-83.1)$ & $56.0(21.4-97.0)$ & $60.0(26.8-89.8)$ & $64.9(34.2-99.7)$ & $101.1(59.7-152.3)$ & 0.0001 \\
\hline Processed meat (g/day) & $20.3(8.6-41.1)$ & $23.9(8.6-36.6)$ & $24.9(8.5-42.4)$ & $25.6(12.4-44.6)$ & $26.0(8.1-42.6)$ & 0.22 \\
\hline $\mathrm{P}: \mathrm{S}$ ratio & $0.49(0.35-0.64)$ & $0.46(0.33-0.58)$ & $0.46(0.37-0.62)$ & $0.46(0.34-0.62)$ & $0.47(0.36-0.59)$ & 0.27 \\
\hline Iron (mg/day) & $10.5(8.9-12.9)$ & $11.2(9.5-13.9)$ & $11.1(9.1-13.6)$ & $11.3(9.0-14.1)$ & $10.5(8.5-13.4)$ & 0.16 \\
\hline Magnesium (mg/day) & $268.2(226.0-3236.4)$ & $278.9(242.1-335.2)$ & $277.3(233.5-338.5)$ & $271.1(231.3-314.3)$ & $266.5(221.7-313.8)$ & 0.23 \\
\hline Fibre (mg/day) & $14.0(10.6-17.9)$ & $14.4(11.8-17.3)$ & $13.9(10.9-17.9)$ & $13.6(11.4-16.9)$ & $13.2(10.3-16.3)$ & 0.26 \\
\hline
\end{tabular}

Values are medians and interquartile ranges

${ }^{a}$ Difference across groups (Kruskall-Wallis)

$P: S$ ratio, polyunsaturated:saturated fat ratio

raised ferritin (group 5). Plasma vitamin $\mathrm{C}$ and adiponectin levels were significantly lower across categories of increasing ferritin concentration. In contrast, concentrations of the hepatic enzymes ALT and GGT increased across the ferritin categories. CRP, fibrinogen and IL-6 did not differ significantly across ferritin categories. Among dietary factors, consumption of red meat was significantly higher with increasing ferritin concentration.

The correlation between ferritin and inflammatory markers was weak (Table 2). In contrast, there was a strong positive correlation between hepatic enzymes and ferritin, and a strong inverse association between adiponectin and baseline serum ferritin.

The distribution of case and control participants among the five groups of baseline ferritin, and the multivariate logistic regression analyses are shown in Table 3. Compared with the lowest quartile of ferritin (group 1) there was a sevenfold higher risk of incident diabetes in the clinically raised ferritin group (group 5) in analyses adjusted for age, sex and BMI. Adjusting for known risk factors and dietary

Table 2 Pearson's correlation coefficients of serum ferritin (ln-transformed) with inflammatory factors, liver function tests and adiponectin in case participants, control participants and all participants: the EPIC diabetes nested case-control study (1993-2000)

\begin{tabular}{|c|c|c|c|c|c|c|}
\hline & \multicolumn{2}{|c|}{ Case participants } & \multicolumn{2}{|c|}{ Control participants } & \multicolumn{2}{|c|}{ All participants } \\
\hline & $r$ & $p$ value & $r$ & $p$ value & $r$ & $p$ value \\
\hline $\mathrm{CRP}^{\mathrm{a}}$ & -0.03 & 0.6 & 0.06 & 0.1 & 0.06 & 0.07 \\
\hline Fibrinogen & 0.04 & 0.5 & -0.07 & 0.1 & -0.02 & 0.5 \\
\hline IL-6 ${ }^{\mathrm{a}}$ & 0.06 & 0.2 & 0.1 & 0.037 & 0.10 & 0.001 \\
\hline $\mathrm{ALT}^{\mathrm{a}}$ & 0.37 & $<0.0001$ & 0.21 & $<0.0001$ & 0.31 & $<0.001$ \\
\hline $\mathrm{GGT}^{\mathrm{a}}$ & 0.29 & $<0.0001$ & 0.29 & $<0.0001$ & 0.33 & $<0.001$ \\
\hline Adiponectin $^{\mathrm{a}}$ & -0.28 & $<0.0001$ & -0.21 & $<0.0001$ & -0.27 & $<0.001$ \\
\hline
\end{tabular}

${ }^{\mathrm{a}} \ln$-transformed 
Table 3 Logistic regression analysis of the association of ferritin and diabetes: the EPIC diabetes nested case-control study (1993-2000)

\begin{tabular}{|c|c|c|c|c|c|c|}
\hline & \multicolumn{5}{|c|}{ Ferritin group } & \multirow[t]{2}{*}{$p$ value } \\
\hline & $\begin{array}{l}\text { Group 1 } \\
\text { (quartile 1) }\end{array}$ & $\begin{array}{l}\text { Group } 2 \\
\text { (quartile 2) }\end{array}$ & $\begin{array}{l}\text { Group } 3 \\
\text { (quartile 3) }\end{array}$ & $\begin{array}{l}\text { Group } 4 \\
\text { (quartile 4) }\end{array}$ & $\begin{array}{l}\text { Group } 5 \\
\text { (raised ferritin) }\end{array}$ & \\
\hline \multicolumn{7}{|l|}{ Ferritin $(\mathrm{ng} / \mathrm{ml})$} \\
\hline Men & $<39.4$ & $39.5-71.1$ & $71.2-112.4$ & 112.5 to $<300$ & $\geq 300$ and $<900$ & \\
\hline Women & $<20.6$ & $20.7-36.7$ & $36.8-63.0$ & 63.1 to $<150$ & $\geq 150$ and $<450$ & \\
\hline Case participants & 72 & 63 & 68 & 110 & 43 & \\
\hline Control participants & 187 & 182 & 187 & 186 & 14 & \\
\hline \multicolumn{7}{|l|}{ OR $(95 \% \mathrm{CI})$ with models adjusted for: } \\
\hline Age, sex, BMI (Model 1) & 1.0 & $0.8(0.5-1.2)$ & $0.9(0.6-1.3)$ & $1.3(0.9-2.0)$ & $7.1(3.6-14.0)$ & $<0.0001$ \\
\hline Model $1+$ family history+physical activity + smoking $(\text { Model } 2)^{\mathrm{b}}$ & 1.0 & $0.8(0.5-1.2)$ & $0.8(0.6-1.3)$ & $1.3(0.9-1.9)$ & $7.5(3.7-15.0)$ & $<0.0001$ \\
\hline Model 2+dietary factors $\left(\right.$ Model 3) ${ }^{\mathrm{c}}$ & 1.0 & $0.9(0.6-1.4)$ & $1.0(0.6-1.5)$ & $1.4(0.9-2.1)$ & $7.4(3.5-15.4)$ & $<0.0001$ \\
\hline Model 3+CRP (Model 4) ${ }^{\mathrm{d}}$ & 1.0 & $0.9(0.6-1.3)$ & $0.9(0.6-1.4)$ & $1.3(0.9-2.0)$ & $6.8(3.2-14.1)$ & $<0.0001$ \\
\hline Model 4+fibrinogen+IL-6 (Model 5) ${ }^{\mathrm{d}}$ & 1.0 & $0.9(0.6-1.4)$ & $1.0(0.6-1.5)$ & $1.3(0.9-2.0)$ & $6.0(2.8-12.8)$ & 0.0005 \\
\hline Model $5+$ LFT $^{\mathrm{e}}$ & 1.0 & $0.8(0.5-1.3)$ & $0.9(0.5-1.4)$ & $1.0(0.7-1.6)$ & $4.0(1.8-9.1)$ & 0.004 \\
\hline Model $5+\mathrm{LFT}^{\mathrm{e}}+$ adiponectin & 1.0 & $0.7(0.5-1.2)$ & $0.7(0.5-1.2)$ & $0.9(0.6-1.5)$ & $3.2(1.3-7.6)$ & 0.007 \\
\hline
\end{tabular}

Age, BMI as continuous variables

${ }^{a}$ Departure from linear trend

${ }^{b}$ Family history coded yes/no; physical activity coded inactive, moderately inactive, moderately active, active; smoking coded never, former or current smoker

${ }^{\mathrm{c}}$ Dietary factors all continuous - total energy intake, alcohol consumption, dietary iron, dietary magnesium, red meat and processed meat intake, plasma vitamin $\mathrm{C}$

${ }^{\mathrm{d}} \mathrm{C}$-reactive protein (CRP, fibrinogen and IL-6 as continuous variables)

${ }^{\mathrm{e}}$ Liver function tests (LFTs) include ALT and GGT as continuous variables; adiponectin as continuous variable

factors increased the risk slightly (odds ratio [OR] 7.4, 95\% CI 3.5-15.4). Adjustment for inflammatory factors slightly attenuated the association but the risk remained markedly elevated (OR 6.0, 95\% CI 2.8-12.8). Further adjustment for hepatic enzymes and adiponectin reduced the risk association but it still remained threefold higher in the highest category of ferritin vs the lowest. Our sensitivity analysis, using alternative upper limit cut-points of 200 or $300 \mathrm{ng} / \mathrm{ml}$ in women, showed that the magnitude and direction of the associations were essentially unchanged. Adjustment for menopausal status in women ( $92 \%$ of case participants and $88 \%$ of control participants were postmenopausal) did not alter the findings.

To reduce the potential confounding effect of obesity we performed all analyses adjusted for BMI, and repeated the analysis with waist circumference in the models, with no change in the magnitude and direction of associations. There was no interaction between BMI and ferritin $(p=0.1)$. We also repeated analyses with more stringent criteria of exclusion of those with ferritin greater than twice the upper limit of normal ( $>600 \mathrm{ng} / \mathrm{ml}$ in men; $>300 \mathrm{ng} / \mathrm{ml}$ in women), without any material change in results. None of the control participants had an $\mathrm{HbA}_{1 \mathrm{c}}$ value $>6 \%$ (control men: $\mathrm{HbA}_{1 \mathrm{c}}$ range $3.5-5.9 \%$, control women: range $3.2-5.9 \%$ ), thus reducing potential bias due to undiagnosed diabetes in the control group. There was no evidence for statistical interaction between ferritin and any of the inflammatory markers.
As there was a suggestion that the associations were non-linear, with statistically significant associations restricted to the highest ferritin category (Table 3), we repeated the analyses for the effect of clinically raised ferritin (Group 5) against all other categories combined (ferritin, groups 1-4), and found a similar magnitude of risk associations for all models as with the comparison of the two extreme ferritin categories (Fig. 1).

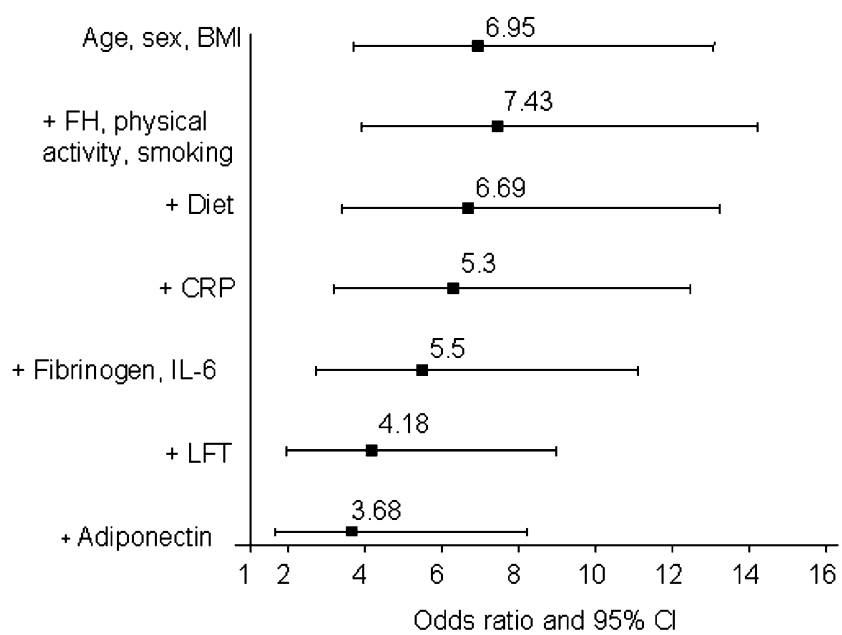

Fig. 1 Odds ratios and $95 \%$ CIs for the association of clinically raised ferritin (group 5) vs ferritin in the normal range (groups 1-4) with incident diabetes in men and women, with adjustment for factors as stated (described in methods). $F H$, family history; $L F T$, liver function tests (ALT and GGT) 


\section{Discussion}

In this prospective study we found a strong association between clinically raised ferritin, below the range indicative of clinical haemochromatosis, and development of incident diabetes. The risk was elevated sevenfold in the clinically raised ferritin group compared with the lowest ferritin category. This striking association is of potential importance in understanding the aetiology of type 2 diabetes.

Our study adds new information concerning the association of serum ferritin and incident type 2 diabetes. Unlike previous studies $[1,2]$ we were able to study the association in both men and women in the same prospective study. There were strong associations of clinically raised ferritin with diabetes, when compared with the whole group of those with normal ferritin, and also when compared with those in the lowest quartile of the ferritin distribution. Our sensitivity analysis showed that this was the case regardless of the cut-point of the upper limit of ferritin in women, in whom there is debate about whether the threshold should be 150 [3], $200[20,21]$ or the same as in men at $300 \mathrm{ng} / \mathrm{ml}$. We found that elevated ferritin was predictive of diabetes independently of a comprehensive range of risk factors and confounders, where previously only a limited number of confounders were accounted for. Specifically, we found that elevated ferritin was associated with diabetes independently of known risk factors for diabetes (age, BMI, sex, family history, physical inactivity and smoking), as well as dietary factors and alcohol intake measured by food diary. We were able to account for the potential confounding effect of three markers of inflammation (IL-6 and fibrinogen in addition to CRP, which was measured in previous studies). In addition, we also accounted for the potential confounding effect of hepatic enzymes and adiponectin, both of which are now known to be associated with incident diabetes, but their association with ferritin is unclear. Data on fasting glucose are not available in the EPIC-Norfolk study, but we suggest that as non-diabetic hyperglycaemia is likely to be on the causal pathway, it is not likely to be a confounder of the ferritin-diabetes association.

The mechanism for the association between ferritin and type 2 diabetes is not established, but iron deposition in the liver may cause insulin resistance by interfering with the ability of insulin to suppress hepatic glucose production [5]. Iron is auto-oxidised to form highly reactive, lipid-soluble iron-oxygen complexes. These free radicals are powerful pro-oxidants, which can change membrane properties and result in tissue damage $[22,23]$. Oxidative stress can also lead to hyperglycaemia through disturbed glucose metabolism [24]. In addition, iron accumulation in hepatocytes may interfere with the insulin-extracting capacity of the liver [25], and affect insulin synthesis and secretion in the pancreas [26]. Iron excess probably contributes initially to insulin resistance and subsequently to decreased insulin secretion [26].

The independent association of hepatic enzymes and incident diabetes has now been well described [10-13]. We found a strong positive correlation between ferritin and the hepatic enzymes, ALT and GGT ( $r$ ranging between 0.29 and $0.37, p<0.0001)$. This is consistent with the recently reported correlation of $0.37(p<0.001)$ between ALT and serum ferritin among 959 women in a cross-sectional Korean study [27]. It is plausible that iron overload in the liver damages hepatocytes, which in turn would result in elevated transaminases and GGT. Although we did not have access to liver ultrasound or biopsy in our study, a correlation between ferritin level and non-alcoholic fatty liver disease or non-alcoholic steatohepatitis, which is associated with elevated ALT, has been described by others in both obese [28] and mainly non-obese [29] individuals. An association between raised hepatic iron concentration and severity of fibrosis in non-alcoholic steatohepatitis has also been reported [30]. Further, Iwasaki et al. also recently described an association between hepatic fat content as measured by computed tomography and ferritin concentration among diabetic individuals [31].

Although it is well described that hypoadiponectinaemia is predictive of future diabetes $[8,9]$, there are no previous published reports of an association between serum ferritin and adiponectin levels. We report a strong negative correlation between adiponectin and ferritin $(r-0.21$ to $-0.28, p<0.0001)$. Although the mechanism underlying this association is not clear, it is plausible that it works through the recently described association of ferritin with abdominal fat [31]. Thus with our observation of the association between ferritin and diabetes, as well as the association between each of hepatic enzymes (representing hepatic steatosis) and adiponectin with ferritin on the one hand and with diabetes on the other, we believe it is a strength of our study to have accounted for these confounders in our analyses.

Limitations of our study merit consideration. We measured serum ferritin as the only marker of iron storage, but did not measure other markers of iron overload such as transferrin saturation, derived from serum iron concentration and total iron-binding capacity. Although a diagnosis of haemochromatosis is made with combined information on ferritin, transferrin saturation, ALT, liver biopsy and genetic mutations for haemochromatosis, we attempted to exclude the possibility of haemochromatosis in our study by excluding those participants who had ferritin elevated more than threefold above the upper limit of normal. We further applied more stringent criteria by excluding from analysis those who had ferritin levels greater than twice the upper limit of normal, with unaltered findings. Our approach of quartiles among the normal range and the 
clinically raised ferritin group excluding those with very high levels, offers a clinical utility that highlights that it is those with ferritin elevated above the normal range who are at greatest risk of developing diabetes. Such an approach was not taken by previous studies as they reported results for quintiles of the ferritin distribution [2], whereby the top quintile included those with levels both below and above the clinically raised level. We have not been able to exclude the possibility that a small number of people with elevated ferritin may have developed haemochromatosis-related diabetes rather than type 2 diabetes, but this is unlikely in the absence of marked hyperferritinaemia, which we were careful to exclude. A further limitation is that we could not specifically measure the known non-genetic causes for iron accumulation, including transfusion iron overload or defective erythropoiesis. Iron overload of undetermined origin includes alcoholic liver disease, non-alcoholic fatty liver disease and chronic hepatitis $\mathrm{C}$ infection, although the mechanisms for iron deposition in these conditions is unknown. We did not screen for hepatitis $\mathrm{C}$ status, but we would expect very low prevalence in this low-risk population as suggested by the estimated prevalence of between 0.55 and $0.7 \%$ in England and Wales in 19911996 [32]. We did, however, measure and include in our analyses both alcohol intake and two measures of hepatic function that are markers for non-alcoholic fatty liver disease. Finally, it should be noted that, for pragmatic reasons, in this large cohort study of $>25,000$ persons, we could only ascertain diagnosed cases of diabetes, thus undiagnosed diabetes was not ascertained biochemically, which would have the overall effect of smaller numbers of cases. We can, however, be reasonably confident that we have minimised the main concern of a potential misclassification of true case participants as control participants in instances of undiagnosed diabetes or failure to self-report diabetes. We can confirm that none of the controls had an $\mathrm{HbA}_{1 \mathrm{c}}$ value $>6 \%$ (control men: $\mathrm{HbA}_{1 \mathrm{c}}$ range 3.5-5.9\%, control women: range $3.2-5.9 \%$ ), thus reducing potential bias due to undiagnosed diabetes in the control group. Our case ascertainment of diagnosed cases was thorough, with information on selfreport supplemented by information from external sources such as general practice records and hospital records as well as death certificates. Our validation of self-report against medical records in 12 of the general practices enabled accurate case ascertainment of clinically diagnosed diabetes.

Our study has several strengths, with its prospective design, inclusion of both men and women, large sample size, and adjustment for a comprehensive battery of risk factors and confounders including measurement of dietary factors by a 7-day food diary. Previous validation studies on this cohort demonstrated that dietary diaries were superior to other methods of assessment of dietary intake [33, 34]. A strength of our study is that as excessive dietary iron intake is a potential reason for variation in iron stores, we adjusted our analyses for dietary iron-related variables (total energy intake, iron intake, and red and processed meat intake), as well as plasma vitamin $\mathrm{C}$, which affects iron absorption, and found that ferritin was associated with diabetes independently of these dietary factors. As ferritin is also an acute-phase reactant and as such reflects not only body iron stores but also subclinical inflammation, we tried to minimise potential confounding by inflammation by adjusting our analyses for three markers of inflammation (CRP, fibrinogen and IL-6), thus making our study more robust than a previous study that adjusted for CRP alone [2]. We were rigorous in our attempts to account for the potential confounding effect of obesity by adjusting all analyses for BMI, and by also adjusting for central obesity (waist circumference).

In conclusion, we have shown prospectively that even modestly elevated ferritin, below levels diagnostic of haemochromatosis or other iron-storage disorders, predicts incident diabetes independently of known risk factors and confounders. These findings have implications for increasing our understanding of the aetiology of type 2 diabetes and merit further study in future studies that help to clarify causality and advance this area of research.

Acknowledgements The EPIC-Norfolk cohort is supported by grant funding from Cancer Research UK, the Medical Research Council, the Stroke Association, the British Heart Foundation, the Department of Health, the Commission of the European Union's Europe against Cancer Programme, the Food Standards Agency, the Department for Environment, Food and Rural Affairs, and the World Health Organization.

Duality of interest None of the authors reports a duality of interest.

\section{References}

1. Salonen JT, Tuomainen TP, Nyyssonen K et al (1998) Relation between iron stores and non-insulin dependent diabetes in men: case-control study. BMJ 317:727

2. Jiang R, Manson JE, Meigs JB et al (2004) Body iron stores in relation to risk of type 2 diabetes in apparently healthy women. JAMA 291:711-717

3. Ford ES, Cogswell ME (1999) Diabetes and serum ferritin concentration among US adults. Diabetes Care 22:1978-1983

4. Tuomainen TP, Nyyssonen K, Salonen R et al (1997) Body iron stores are associated with serum insulin and blood glucose concentrations. Population study in 1,013 eastern Finnish men. Diabetes Care 20:426-428

5. Fernandez-Real JM, Ricart-Engel W, Arroyo E et al (1998) Serum ferritin as a component of the insulin resistance syndrome. Diabetes Care 21:62-68

6. Hughes K, Choo M, Kuperan P et al (1998) Cardiovascular risk factors in non-insulin-dependent diabetics compared to nondiabetic controls: a population-based survey among Asians in Singapore. Atherosclerosis 136:25-31

7. Acton RT, Barton JC, Passmore LV et al (2006) Relationships of serum ferritin, transferrin saturation, and HFE mutations and self- 
reported diabetes in the Hemochromatosis and Iron Overload Screening (HEIRS) study. Diabetes Care 29:2084-2089

8. Lindsay RS, Funahashi T, Hanson RL et al (2002) Adiponectin and development of type 2 diabetes in the Pima Indian population. Lancet 360:57-58

9. Duncan BB, Schmidt MI, Pankow JS et al (2004) Adiponectin and the development of type 2 diabetes: the atherosclerosis risk in communities study. Diabetes 53:2473-2478

10. Sattar N, Scherbakova O, Ford I et al (2004) Elevated alanine aminotransferase predicts new-onset type 2 diabetes independently of classical risk factors, metabolic syndrome, and C-reactive protein in the west of Scotland coronary prevention study. Diabetes 53:2855-2860

11. Hanley AJ, Williams K, Festa A et al (2004) Elevations in markers of liver injury and risk of type 2 diabetes: the insulin resistance atherosclerosis study. Diabetes 53:2623-2632

12. Nakanishi N, Suzuki K, Tatara K (2004) Serum gamma-glutamyltransferase and risk of metabolic syndrome and type 2 diabetes in middle-aged Japanese men. Diabetes Care 27:1427-1432

13. Lee DH, Silventoinen K, Jacobs DR Jr et al (2004) Gammaglutamyltransferase, obesity, and the risk of type 2 diabetes: observational cohort study among 20,158 middle-aged men and women. J Clin Endocrinol Metab 89:5410-5414

14. Fumeron F, Pean F, Driss F et al (2006) Ferritin and transferrin are both predictive of the onset of hyperglycemia in men and women over 3 years: the data from an epidemiological study on the Insulin Resistance Syndrome (DESIR) study. Diabetes Care 29:2090-2094

15. Day N, Oakes S, Luben R et al (1999) EPIC-Norfolk: study design and characteristics of the cohort. European Prospective Investigation of Cancer. Br J Cancer 80(Suppl 1):95-103

16. Harding AH, Day NE, Khaw KT et al (2004) Dietary fat and the risk of clinical type 2 diabetes: the European prospective investigation of Cancer-Norfolk study. Am J Epidemiol 159:73-82

17. Wareham NJ, Jakes RW, Rennie KL et al (2003) Validity and repeatability of a simple index derived from the short physical activity questionnaire used in the European Prospective Investigation into Cancer and Nutrition (EPIC) study. Public Health Nutr 6:407-413

18. Welch AA, McTaggart A, Mulligan AA et al (2001) DINER (Data Into Nutrients for Epidemiological Research) - a new dataentry program for nutritional analysis in the EPIC-Norfolk cohort and the 7-day diary method. Public Health Nutr $4: 1253-1265$
19. Bingham SA, Welch AA, McTaggart A et al (2001) Nutritional methods in the European prospective investigation of cancer in Norfolk. Public Health Nutr 4:847-858

20. Jehn M, Clark JM, Guallar E (2004) Serum ferritin and risk of the metabolic syndrome in US adults. Diabetes Care 27:2422-2428

21. Fairbanks VF (1991) Laboratory testing for iron status. Hosp Pract (Off Ed) 26(Suppl 3):17-24

22. Oberley LW (1988) Free radicals and diabetes. Free Radic Biol Med 5:113-124

23. Wolff SP (1993) Diabetes mellitus and free radicals. Free radicals, transition metals and oxidative stress in the aetiology of diabetes mellitus and complications. Br Med Bull 49:642-652

24. Opara EC (2004) Role of oxidative stress in the etiology of type 2 diabetes and the effect of antioxidant supplementation on glycemic control. J Investig Med 52:19-23

25. Niederau C, Berger M, Stremmel W et al (1984) Hyperinsulinaemia in non-cirrhotic haemochromatosis: impaired hepatic insulin degradation? Diabetologia 26:441-444

26. Wilson JG, Lindquist JH, Grambow SC et al (2003) Potential role of increased iron stores in diabetes. Am J Med Sci 325:332-339

27. Choi KM, Lee KW, Kim HY et al (2005) Association among serum ferritin, alanine aminotransferase levels, and metabolic syndrome in Korean postmenopausal women. Metabolism 54:1510-1514

28. Hsiao TJ, Chen JC, Wang JD (2004) Insulin resistance and ferritin as major determinants of nonalcoholic fatty liver disease in apparently healthy obese patients. Int J Obes 28:167-172

29. Bacon BR, Farahvash MJ, Janney CG et al (1994) Nonalcoholic steatohepatitis: an expanded clinical entity. Gastroenterology 107:1103-1109

30. George DK, Goldwurm S, MacDonald GA et al (1998) Increased hepatic iron concentration in nonalcoholic steatohepatitis is associated with increased fibrosis. Gastroenterology 114:311-318

31. Iwasaki T, Nakajima A, Yoneda $M$ et al (2005) Serum ferritin is associated with visceral fat area and subcutaneous fat area. Diabetes Care 28:2486-2491

32. Balogun MA, Ramsay ME, Hesketh LM et al (2002) The prevalence of hepatitis C in England and Wales. J Infect 45:219-226

33. Bingham SA, Gill C, Welch A et al (1994) Comparison of dietary assessment methods in nutritional epidemiology: weighed records v. $24 \mathrm{~h}$ recalls, food frequency questionnaires and estimated diet records. Br J Nutr 74:619-643

34. Bingham SA, Cassidy A, Cole $T$ et al (1995) Validation of weighed records and other methods of dietary assessment using the $24 \mathrm{~h}$ urine nitrogen technique and other biological markers. Br J Nutr 73:531-550 